\title{
Enduring Challenge of Latent Tuberculosis in Older Nursing Home Residents: A Brief Review
}

\author{
Asif Khan ${ }^{\mathrm{a}, \mathrm{b}}$, Anh Rebhan ${ }^{\mathrm{a}}$, Donna Seminara ${ }^{\mathrm{a}}$, Anita Szerszen ${ }^{\mathrm{a}}$
}

\begin{abstract}
Tuberculosis (TB) kills almost 4,000 people a day and is competing with human immunodeficiency virus/acquired immune deficiency syndrome (HIV/AIDS) as the most deadly infectious disease in the world. The gold standards of detection and management of latent tuberculosis infection (LTBI) have not been successful in complete eradication of the disease. Current screening modalities of TB include tuberculin skin testing (TST) and/or interferon $-\gamma$ release assay (IGRA). However, these screening tests have been heavily studied in healthy populations but not in the elderly who are more likely to have multiple risk factors for progression to active TB from LTBI. The largest population that is harboring LTBI is the elderly, specifically those residing in nursing homes. Yet, unfortunately, guidelines for standards of detection and treatment for this specific group are lacking. In this review, we look at TST versus IGRA screening for LTBI in the elderly living in nursing homes. We review a cross-sectional study done at Staten Island University Hospital, and several other assessments of the sensitivity and accuracy of both screening tools. Furthermore, this review looks at the appropriateness of current LTBI treatment and prophylaxis in elderly patients residing in close quarters. The reviews point to the superiority of IGRA testing in the elderly for screening LTBI. The IGRA has been shown to be more sensitive to the detection of LTBI than TST. Additionally, medical complexities that the elderly population possesses may present challenges and resistance to standard treatments of LTBI. It is recommended via the literature that the addition of vitamin $\mathrm{D}$, or alternative therapies (e.g. rifampin) could produce better outcomes for elderly patients with LTBI than the current 9 months of isoniazid (INH). As the older adults represent the fastest growing segment of our population and the largest LTBI reservoir in the USA, revisiting screening and treatment of LTBI in the elderly living in nursing homes may prove to lead to a path of TB eradication once and for all.
\end{abstract}

Keywords: Screening tuberculosis; Latent tuberculosis infection; Tuberculin skin testing; Interferon- $\gamma$ release assay; Nursing home

Manuscript submitted February 4, 2019, accepted March 4, 2019

aDepartment of Internal Medicine, Staten Island University Hospital, Staten Island, NY, USA

${ }^{b}$ Corresponding Author: Asif Khan, Department of Internal Medicine, Staten Island University Hospital, 475 Seaview Ave, Staten Island, NY 10305, USA. Email: akhan25@northwell.edu

doi: https://doi.org/10.14740/jocmr3763

\section{Introduction}

One of the oldest human disease known, infecting our ancestors over 20,000 years ago, tuberculosis (TB) continues to be a diagnostic and therapeutic challenge even in the 21 st century. TB kills almost 4,000 people a day and is competing with human immunodeficiency virus/acquired immune deficiency syndrome (HIV/AIDS) as the most deadly infectious disease in the world [1]. In economically developed nations, nearly 380 million people are infected with Mycobacterium tuberculosis $[2,3]$. More than 11 million in the USA are found to have latent tuberculosis infection (LTBI), which carries a $5-10 \%$ lifetime risk of progression into active disease [2].

Older adults remain by far the largest TB reservoir [4] in the USA, as almost one-quarter of all TB cases are found among those 65 years of age and older [5]. A progressive increase in life expectancy and exponentially growing numbers of the very old present special and unexpected challenges to TB control, particularly among skilled nursing facilities. Despite advances in diagnosis and treatment, the rate of TB is four times higher in residents of nursing homes than in the general population [6]. It is estimated that 1.8 million Americans live in nursing homes, the majority of whom $(88 \%)$ are elderly. As they represent a disproportionate share of TB-associated mortality [3], an accurate identification of risk factors leading to TB reactivation, and timely LTBI detection and treatment deserve particular attention.

\section{Risk Factors for Reactivation of LTBI in Elderly Nursing Home Residents}

Widely recognized risk factors for LTBI include immigration from endemic countries, work in health-care industry, imprisonment, homelessness, and illicit drug use. For the residents of skilled nursing facilities, in addition to past TB exposures as a consequence of the above factors, the age-associated immune senescence, the use of immuno-modulating drugs, their co-morbid illnesses, protein malnutrition and close proximity between residents further elevate the risk of TB infection and reactivation of latent disease [7]. Immunocompromised older patients have increased risk of developing extra-pulmonary TB that can involve any organ (gastrointestinal tract, central nervous system, etc.) and diagnosis may be missed as symptoms mimic other conditions.

Immunodeficiency places older nursing home patients at 
higher risk for LTBI reactivation. HIV/AIDS is a well-recognized immunodeficiency state. What is less known is that, with medical advancement, people with HIV are expected to live almost as long as healthy individuals $[8,9]$ and that $17 \%$ of all new HIV diagnoses are made among persons over 50 years old [10]. In fact, the Centers for Disease Control (CDC) estimated that $45 \%$ of Americans living with HIV were aged 50 and older in 2014 [11]. With steadily increasing life expectancy some of these patients will be found among residents of skilled nursing facilities. While for healthy persons the lifetime risk of LTBI progressing to active $\mathrm{TB}$ is $5-10 \%$, in $\mathrm{HIV}^{+}$patients, this is their annual risk (5-10\%) [2]. Furthermore, extra-pulmonary symptoms develop over the course of many years in non-HIV infected patients. However, patients with HIV, especially those older and frail, can progress to active disease within weeks to months [2].

There is very little literature for $\mathrm{CD}^{+}$cut-off established for TB prophylaxis in persons with HIV. Older adults are not routinely screened for HIV. It is therefore imperative that TB surveillance with the best sensitive and specific screening measures should be held high throughout a patient's residence at a nursing home, for all patients, not just for those with known HIV [12].

The same holds true for organ transplant recipients who are surviving well into advanced age, often under immunosuppressive therapy. Immune-modulating therapy may also be offered to nursing home residents suffering from collagen vascular diseases, like rheumatoid arthritis. As tumor necrosis factor $\alpha(\mathrm{TNF}-\alpha)$ antagonists increase risk for reactivation of LTBI, current guidelines recommend screening prior to initiation of such therapies $[13,14]$, but not for corticosteroids. Yet, adults treated with high doses of prednisone ( $>15 \mathrm{mg}$ /day) for only 2 - 4 weeks are already at increased risk for LTBI reactivation [15]. Conditions requiring the use of glucocorticoids are common in nursing home populations, including asthma, chronic obstructive pulmonary disease (COPD), gout, to mention a few. Some nursing home residents, such as those with advanced dementia and asthma or COPD may be given an oral corticosteroid as an alternative to inhalers if they are unable to use them.

Silicosis is a rarely diagnosed condition in older adults that may require corticosteroids. Silicosis and prolonged occupational dust exposure, even without overt disease, confer a risk for pulmonary $\mathrm{TB}$ up to 40 times higher than that in healthy controls [16]. While we are astute about screening for LTBI in patients coming from high TB prevalence areas, occupational history or potential for dust exposure is rarely assessed in older residents of nursing home. Rates of silicosis are declining in the USA, yet occupational history should be elicited especially for residents from developing countries and regions known for mining where silicosis may be more prevalent, such as India, Brazil, Chile, Turkey, Poland, Ukraine, and in the USA: West Virginia, Nevada, and Michigan [17]. This is an important risk to emphasize in education of physicians treating older adults.

Another risk factor for reactivation of LTBI in older nursing home residents is chronic renal failure (CKD), especially when it requires hemodialysis [18]. Their risk of TB infection increases an estimated 6.9 to 52.5 times [19]. Furthermore, dialysis patients are also more likely to be institutionalized in skilled nursing facilities. Both physicians and nursing staff should be aware about the non-specific presentation of TB in older adults with CKD and inclusion of TB in the differential diagnosis in CKD patients exhibiting constitutional symptoms such as anorexia, fever, malaise, and weight loss [19]. Extrapulmonary forms of TB predominate in these patients, which requires high index of suspicion as the prognosis is dependent on early diagnosis and treatment. With missed signs and symptoms, the incidence of TB in CKD has risen to $4 \%$ in recent years [20]. Tuberculin skin testing (TST) was also found to be negative in the majority of those patients [20]. The immunocompromised state of being on hemodialysis blurs the typical presentation of TB and augments TB tests, with anergy to TST reported in $56 \%$ of patients [18].

TST is also affected by functional waning of the immune system known as immunosenescence. As a consequence of normal aging, immunosenescence underlies the compromised protection against TB in older patients. Both innate (dendritic cells, natural killer cells, macrophages and complement system) and adaptive immune responses (lymphoid progenitors, $\mathrm{B}$ cells and T cells) are affected [21]. In older adults, this is manifested by an increased susceptibility to infections, particularly of respiratory tract, lower vaccine efficacy, and increased autoimmunity, cancer and other chronic diseases [22, 23]. In the context of TB, diminished T-cell response and T-cellmacrophage interactions are clinically manifested as altered delayed-type hypersensitivity reactions [24]. The progressive thymic involution [25] and reduced proliferative capacity of the hematopoietic stem cells [26] are considered to be the main mechanisms by which the pool of $\mathrm{CD}^{+}$and $\mathrm{CD} 8^{+}$cells decline with age. This hampers the availability of TB-specific $\mathrm{CD}^{+}$cells at the site of infection and explains why elderly patients often fail to mount an appropriate immune response and have increased susceptibility for TB [27].

One of the keys to a healthy immune system is healthy nutrition. Sadly, nutritional deficiencies are present in one-third of the elderly in developed countries [28]. It has been well established that vitamin $\mathrm{D}$ is an essential micronutrient and plays a beneficial role in maintaining a healthy immune system. Prior to the introduction of the antibiotic era, the antimicrobial qualities of vitamin $\mathrm{D}$, by modulating the innate immune response, were utilized for the treatment of TB [29]. Active TB patients were treated with cod liver oils and prolonged sun exposure $[30,31]$. The active form of vitamin $\mathrm{D}\left(1,25(\mathrm{OH})_{2} \mathrm{D}_{3}\right)$ leads to activation of cathelicidin, a microbicidal peptide for Mycobacterium tuberculosis [32]. Patients with vitamin D deficiency $\left(1,25(\mathrm{OH})_{2} \mathrm{D}_{3}\right)$ have been shown to have increased susceptibility to $\mathrm{TB}$, thus enticing the consideration of vitamin $\mathrm{D}$ as an anti-TB therapy [33]. Vitamin D deficiency increases the risk of TB and is associated with a 5-fold increased risk for progression to active TB in those with LTBI [34]. Currently, there are ongoing trials assessing the role of vitamin D supplementation in TB prophylaxis.

As the population is aging, diabetes mellitus (DM) has reemerged as a risk factor for TB. While incidence of TB has declined in the developed world, there has been a global rise in DM rates particularly due to the increasing epidemic of obesity, particularly in lower socioeconomic regions [35]. A recent meta-analysis with over 1 million participants supported 
prior studies of DM increasing risk of active and LTBI (relative risk 3.11, 95\% confidence interval (CI) 2.27 - 4.26) [36]. Within nursing homes, increasing numbers of elderly residents live with a diagnosis of $\mathrm{DM}$, with prevalence ranging from $25-34 \%$ [37]. Poor DM control has been associated with an increased risk for active TB, as compared to those with hemoglobin $\mathrm{A} 1 \mathrm{c}(\mathrm{HgA} 1 \mathrm{c})$ of less than 7 [38]. Hyperglycemia may affect the immunity to TB with a potential for a diminished response to treatment as manifested by a culture conversion delay and a higher rate of relapse in diabetic patients treated for TB. Given the adverse consequences of hypoglycemia in frail patients with DM under tight glycemic control, the prevention and treatment of TB pose a clinical challenge in this patient population.

Therefore, in the context of these vulnerable populations, LTBI screening should be tailored to the severity and number of identified risk factors in individual patients.

\section{Detecting LTBI}

LTBI can be identified by a positive reaction to the TST or a positive interferon- $\gamma$ release assay (IGRA) [2]. The USA could save an estimated $\$ 60$ to 90 million annually through detection of LTBI as it would prevent the 9,000 to 10,000 newly diagnosed cases of active TB [39].

In 1990, the CDC recommended the use of the two-step TST approach as the initial screening test for LTBI in all residents of nursing homes and long-term care centers, upon admission. This was supplemented with an annual one-step TST in high prevalence areas [6]. In 2013, CDC guidelines were updated to include the use of IGRA that indirectly measures the host immune response to specific Mycobacterium peptides. Neither an IGRA nor a TST can distinguish LTBI from active TB, which may contribute to the under-detection of TB in these populations.

\section{TST in the Elderly}

TST involves the intradermal injection of purified protein derivative (PPD), which leads to a delayed-type hypersensitivity response that peaks at $48-72 \mathrm{~h}$, causing a cutaneous induration at the site of injection [8]. As the TST depends on normal T-cell function, an attenuated T-cell response due to immune senescence, malnutrition and immunocompromise, may affect the sensitivity to detect LTBI. A positive TST result may indicate an increased risk of developing or currently having active TB.

It must be noted that there is a potential of $30 \%$ false negative detection rate in immunocompetent patients. Thus, a negative skin test does not exclude the diagnosis of TB [6]. In men, TST reactivity decreases from $50 \%$ in patients $65-74$ years of age to $10 \%$ for those older than 95 . In women, the reactivity rate is even lower and decreases from $40 \%$ in patients 65 - 75 years old to about $5 \%$ at age 95 or older. Conversely, due to tuberculin's cross reactivity with the environmental mycobacteria and Mycobacterium bovis Bacillus Calmette-Guerin
(BCG) vaccine, TST is also prone to false positive results and therefore lacks specificity [5]. Errors in placement and 13-15\% variability in reading of the skin test can also yield false-positive or false-negative results [40].

Cutaneous delayed hypersensitivity response was investigated using TST in elderly nursing home residents, geriatric age clinic patients, and was compared to healthy young adults. Anergy was observed in $34 \%$ of nursing home subjects, $17 \%$ of geriatric clinic patients, and was negligible in young people [10]. It is interesting to note, that a qualitative and quantitative decline in cell-mediated immunity was seen, with significantly lower mean and maximal induration in nursing home residents as compared to the other groups [10]. To increase the sensitivity of TST, a skin anergy panel using mumps and candida antigens was performed to distinguish between the negative TST result and lack of response to tuberculin due to diminished cellular response [41]. However, because of its poor reproducibility and inadequate standardization, anergy testing is no longer recommended [42].

Not all nursing homes are screening for LTBI with annual TST following a negative two-step test. It has been well documented that patients who had repeatedly negative TST have developed disseminated TB after administration of immunosuppressive therapy [43]. Immunosuppression, either due to therapy or underlying conditions, like DM, in context of the age-associated immune senescence makes a correct diagnosis quite challenging [7]. Therefore, the two-step TST may not be sensitive enough to detect latent TB in elderly patients.

\section{IGRAs in the Elderly}

The introduction of IGRAs using specific Mycobacterium tuberculosis antigens for immune-diagnosis is an important step towards improved detection of latent TB. IGRA is an ex vivo blood test identifying and quantifying interferon- $\gamma$ (IFN- $\gamma$ ) from stimulated T lymphocytes with tuberculin PPDs. They have superior specificity and equal, if not better, sensitivity compared to TST in adults [44-47]. There are currently two FDA approved IGRAs available in the USA: QuantiFERONTB Gold-in-Tube test and T-SPOT.TB test. Both types of IGRA are indirect measures of host immune responses to specific Mycobacterium peptides ESAT-6 and CFP-10. The number of anti-mycobacterial effector T cells releasing IFN- $\gamma$ is quantified using these assays. Because more specific peptides are used (not present in BCG or non-tuberculous mycobacteria), it is thought to be more specific for detection of latent $\mathrm{TB}$ [48]. The T-SPOT.TB test does not suffer from the "boosting" phenomenon that can affect the TST, as there is no injection of immunogenic substances into the body.

\section{TST Versus IGRA}

There are a few advantages of the newer IGRAs over TST. IGRA is a one-time blood test, circumventing the need for follow-up, and results are readily available within $24 \mathrm{~h}$. IGRAs are unaffected by BCG vaccination and are at least as sensitive 
as the TST for detecting active TB. Concurrent administration of live vaccine and TST or IGRA testing may cause false negative results due to decreased response to the mycobacterium antigen. It is recommended to wait at least 4 weeks before giving a routine TST or IGRA [49].

In a German study both sensitivity and specificity of TSPOT.TB were evaluated and compared to those of TST. Out of the 90 patients studied, 72 patients with diagnosed pulmonary or extra-pulmonary $\mathrm{TB}$, the diagnostic sensitivity reached $97 \%$ with T-SPOT.TB. A total of $89 \%$ of selected subgroup patients were positive in the TST compared to $100 \%$ in the TSPOT.TB test $(\mathrm{P}=0.056)$. T-SPOT.TB was negative for $92 \%$ of the 12 patients in whom active TB disease was ruled out [50].

In 2001, Mazurek et al studied 1,226 adults (mean age, 39 years) with varying risks of Mycobacterium tuberculosis infection or suspected active TB, to compare and investigate any potential discordance between IFN- $\gamma$ assay and TST. The results of screening were stratified across four groups of varying risk level: low risk, high risk of LTBI, suspected TB, and prior culture-confirmed TB. Overall agreement between the IFN- $\gamma$ assay and the TST was $83.1 \%$. In the high-risk group, there was an $11 \%$ discordance rate among negative TST results. The discordance proportion increased to $21 \%$ in the group suspected to have LTBI [51]. These statistics clearly indicate that individuals who may be infected with latent $T B$ go undetected with skin testing. Another study such as that conducted by Lee et al [52] showed that T-SPOT.TB assay sensitivity (96.6\%) was superior to that of TST (66.7\%); while QuantiFERON Gold assay specificity $(91.6 \%)$ was higher than that of traditional skin testing (78.6\%); the study group included those with known active TB and those with low-risk of TB infection [52].

The above-mentioned studies suggest that a large number of residents of long-term care facilities may not be properly assessed for presence of LTBI due to unreliability of TST in elderly populations with high risk of TB exposure allowing LTBI underestimation and missed opportunities to adequately manage LTBI in vulnerable elders. Current CDC guidelines recommend using both a TST and an IGRA in situations to increase treatment compliance and also in healthy persons with low risk for both infection and disease progression [53]. However, validation is challenging due to the lack of a diagnostic gold standard test. The CDC also recommends IGRA as the preferred method of testing in patients who have a low likelihood of follow-up for TST reading, and persons who received $\mathrm{BCG}$ in the past.

Upon analysis of the existing literature on both traditional and new methodologies of LTBI diagnosis, it is apparent that for elderly patients at high risk for TB exposure (nursing home dwelling individuals), accurate detection of LTBI proves especially challenging. It may be in fact due to a synergy of factors and/or advanced age alone.

\section{Conclusions}

In the USA, TB notifications are increasingly evident among the elderly population (65 years of age and older) [54]. Tech- nological advances in diagnosis have challenged the traditional TST for LTBI diagnosis. Despite advantages of the newer IGRA tests, there is still no new "gold standard" and Mantoux test remains the current practice standard for identifying TB infection [55]. Since nursing homes usually do not conduct annual TST if patients have a negative two-step test on intake, many cases of TB in older adults may go undetected. This decreased sensitivity of TST in older adults is a consequence of the age-associated immune senescence. IGRA tests appear to be more sensitive in the nursing home population, although current CDC guidelines do not delineate the optimal methods of screening for individuals in high risk living environments such as nursing facilities. We recognize that IGRA is an expensive alternative to TST. However, given the health and economic costs of TB in this patient population it may be worthwhile to closely examine the cost/benefit ratio of the methods used to detect LTBI. As the older adults represent the fastest growing segment of our population and the largest LTBI reservoir in the USA, relentless surveillance in individuals at heightened risk, such as nursing home residents, remains a crucial step towards elimination of this ancient disease.

\section{Acknowledgments}

None to declare.

\section{Financial Disclosure}

None.

\section{Conflict of Interest}

The authors declare that they have no conflict of interest.

\section{Author Contributions}

AK retrieved the data, performed the statistical analysis and wrote the manuscript; AR retrieved the data, and wrote the manuscript; DS wrote the manuscript; and AS corrected the manuscript.

\section{References}

1. World Health Organization. Global tuberculosis report 2016. 2016. http://www.who.int. Accessed September 23, 2018.

2. Centers for disease control. TB Fact Sheets. http://www. cdc.gov/tb/pubs/tbfactsheets/QFT.htm. Accessed January 10, 2017.

3. Strausbaugh LJ, Joseph CJ. Epidemiology and prevention of infections in residents of long term care facilities. Infect Control Hosp Epidemiol. 1999; 2nd edition:1461-1482.

4. TB elimination tuberculin skin testing what is it? How 
is the TST administered? How are TST reactions interpreted? Classification of the tuberculin skin test reaction what are false-positive reactions? http://www.cdc.gov/tb. Accessed September 23, 2018.

5. Nisar M, Williams CS, Ashby D, Davies PD. Tuberculin testing in residential homes for the elderly. Thorax. 1993;48(12):1257-1260.

6. Prevention and control of tuberculosis in facilities providing long-term care to the elderly. Recommendations of the Advisory Committee for Elimination of Tuberculosis. MMWR Recomm Rep. 1990;39(RR-10):7-13.

7. Hochberg NS, Rekhtman S, Burns J, Ganley-Leal L, Helbig S, Watts NS, Brandeis GH, et al. The complexity of diagnosing latent tuberculosis infection in older adults in long-term care facilities. Int J Infect Dis. 2016;44:37-43.

8. In: Implementing the WHO Stop TB Strategy: A Handbook for National Tuberculosis Control Programmes. Geneva, 2008.

9. Nasi M, De Biasi S, Gibellini L, Bianchini E, Pecorini S, Bacca V, Guaraldi G, et al. Ageing and inflammation in patients with HIV infection. Clin Exp Immunol. 2017;187(1):44-52.

10. Rodysill KJ, Hansen L, O'Leary JJ. Cutaneous-delayed hypersensitivity in nursing home and geriatric clinic patients. Implications for the tuberculin test. J Am Geriatr Soc. 1989;37(5):435-443.

11. Brooks JT, Buchacz K, Gebo KA, Mermin J. HIV infection and older Americans: the public health perspective. Am J Public Health. 2012;102(8):1516-1526.

12. Kanabus A. Information about tuberculosis. Global Health Education. https://www.tbfacts.org/tb-hiv/. Published 2017.

13. Bongartz T, Sutton AJ, Sweeting MJ, Buchan I, Matteson EL, Montori V. Anti-TNF antibody therapy in rheumatoid arthritis and the risk of serious infections and malignancies: systematic review and meta-analysis of rare harmful effects in randomized controlled trials. JAMA. 2006;295(19):2275-2285.

14. Gardam MA, Keystone EC, Menzies R, Manners S, Skamene E, Long R, Vinh DC. Anti-tumour necrosis factor agents and tuberculosis risk: mechanisms of action and clinical management. Lancet Infect Dis. 2003;3(3):148155.

15. Jick SS, Lieberman ES, Rahman MU, Choi HK. Glucocorticoid use, other associated factors, and the risk of tuberculosis. Arthritis Rheum. 2006;55(1):19-26.

16. Cheepsattayakorn A, Cheepsattayakorn R. Silicosis-associated tuberculosis: management and control. Am J Public Heal Res. 2018;6:125-129.

17. Barboza CE, Winter DH, Seiscento M, Santos Ude P, Terra Filho M. Tuberculosis and silicosis: epidemiology, diagnosis and chemoprophylaxis. J Bras Pneumol. 2008;34(11):959-966.

18. Abdelrahman M, Sinha AK, Karkar A. Tuberculosis in end-stage renal disease patients on hemodialysis. Hemodial Int. 2006;10(4):360-364.

19. Hussein MM, Mooij JM, Roujouleh H. Tuberculosis and chronic renal disease. Semin Dial. 2003;16(1):38-44.

20. Venkata RK, Kumar S, Krishna RP, Kumar SB, Pad- manabhan S, Kumar S. Tuberculosis in chronic kidney disease. Clin Nephrol. 2007;67(4):217-220.

21. DelaRosa O, Pawelec G, Peralbo E, Wikby A, Mariani E, Mocchegiani E, Tarazona R, et al. Immunological biomarkers of ageing in man: changes in both innate and adaptive immunity are associated with health and longevity. Biogerontology. 2006;7(5-6):471-481.

22. Gavazzi G, Herrmann F, Krause KH. Aging and infectious diseases in the developing world. Clin Infect Dis. 2004;39(1):83-91.

23. Sasaki S, Sullivan M, Narvaez CF, Holmes TH, Furman D, Zheng NY, Nishtala M, et al. Limited efficacy of inactivated influenza vaccine in elderly individuals is associated with decreased production of vaccine-specific antibodies. J Clin Invest. 2011;121(8):3109-3119.

24. Aw D, Silva AB, Palmer DB. Immunosenescence: emerging challenges for an ageing population. Immunology. 2007;120(4):435-446.

25. Moro-Garcia MA, Alonso-Arias R, Lopez-Larrea C. Molecular mechanisms involved in the aging of the T-cell immune response. Curr Genomics. 2012;13(8):589-602.

26. Lansdorp PM, Dragowska W, Thomas TE, Little MT, Mayani H. Age-related decline in proliferative potential of purified stem cell candidates. Blood Cells. 1994;20(23):376-380; discussion 380-371.

27. Orme IM, Griffin JP, Roberts AD, Ernst DN. Evidence for a defective accumulation of protective $\mathrm{T}$ cells in old mice infected with Mycobacterium tuberculosis. Cell Immunol. 1993;147(1):222-229.

28. Chandra RK. Nutrition and the immune system: an introduction. Am J Clin Nutr. 1997;66(2):460S-463S.

29. Ellman P, Anderson KH. Calciferol in tuberculous peritonitis with disseminated tuberculosis. $\mathrm{Br}$ Med J. 1948;1(4547):394.

30. Williams CJB. Cod-liver oil in phthisis. Lond J Med. 1849;1(1):1. https://www.ncbi.nlm.nih.gov/pmc/articles/ PMC2543222/. Accessed September 23, 2018.

31. Charpy J. Quelques traitments vitamines ou par substances fonctionelles en dermatologie. Bull Med. 1950;24:505.

32. Holick MF. Vitamin D deficiency. N Engl J Med. 2007;357(3):266-281.

33. Gibney KB, MacGregor L, Leder K, Torresi J, Marshall $\mathrm{C}$, Ebeling PR, Biggs BA. Vitamin D deficiency is associated with tuberculosis and latent tuberculosis infection in immigrants from sub-Saharan Africa. Clin Infect Dis. 2008;46(3):443-446.

34. Talat N, Perry S, Parsonnet J, Dawood G, Hussain R. Vitamin d deficiency and tuberculosis progression. Emerg Infect Dis. 2010;16(5):853-855.

35. Dooley KE, Chaisson RE. Tuberculosis and diabetes mellitus: convergence of two epidemics. Lancet Infect Dis. 2009;9(12):737-746.

36. Jeon CY, Murray MB. Diabetes mellitus increases the risk of active tuberculosis: a systematic review of 13 observational studies. PLoS Med. 2008;5(7):e152.

37. Munshi MN, Florez H, Huang ES, Kalyani RR, Mupanomunda M, Pandya N, Swift CS, et al. Management of diabetes in long-term care and skilled nursing facilities: a position statement of the American Diabetes Association. 
Diabetes Care. 2016;39(2):308-318.

38. Leung CC, Lam TH, Chan WM, Yew WW, Ho KS, Leung GM, Law WS, et al. Diabetic control and risk of tuberculosis: a cohort study. Am J Epidemiol. 2008;167(12):14861494.

39. Khan K, Muennig P, Behta M, Zivin JG. Global drugresistance patterns and the management of latent tuberculosis infection in immigrants to the United States. N Engl J Med. 2002;347(23):1850-1859.

40. Chaparas SD, Vandiviere HM, Melvin I, Koch G, Becker C. Tuberculin test. Variability with the Mantoux procedure. Am Rev Respir Dis. 1985;132(1):175-177.

41. Dorken E, Grzybowski S, Allen EA. Significance of the tuberculin test in the elderly. Chest. 1987;92(2):237-240.

42. Slovis BS, Plitman JD, Haas DW. The case against anergy testing as a routine adjunct to tuberculin skin testing. JAMA. 2000;283(15):2003-2007.

43. Perez-Stable EJ, Flaherty D, Schecter G, Slutkin G, Hopewell PC. Conversion and reversion of tuberculin reactions in nursing home residents. Am Rev Respir Dis. 1988;137(4):801-804.

44. Shams H, Weis SE, Klucar P, Lalvani A, Moonan PK, Pogoda JM, Ewer K, et al. Enzyme-linked immunospot and tuberculin skin testing to detect latent tuberculosis infection. Am J Respir Crit Care Med. 2005;172(9):11611168.

45. Sterling TR, Villarino ME, Borisov AS, Shang N, Gordin F, Bliven-Sizemore E, Hackman J, et al. Three months of rifapentine and isoniazid for latent tuberculosis infection. N Engl J Med. 2011;365(23):2155-2166.

46. Menzies D, Pai M, Comstock G. Meta-analysis: new tests for the diagnosis of latent tuberculosis infection: areas of uncertainty and recommendations for research. Ann Intern Med. 2007;146(5):340-354.
47. Huebner RE, Schein MF, Bass JB, Jr. The tuberculin skin test. Clin Infect Dis. 1993;17(6):968-975.

48. Mazurek GH, Jereb J, Lobue P, Iademarco MF, Metchock B, Vernon A, Division of Tuberculosis Elimination NCfHIVSTD, et al. Guidelines for using the QuantiFERON-TB Gold test for detecting Mycobacterium tuberculosis infection, United States. MMWR Recomm Rep. 2005;54(RR-15):49-55.

49. William A, Wolfe C, Hamborsky J. Epidemiology and prevention of vaccine-preventable diseases. 12th ed. The Public Health Foundation; 2011.

50. Meier T, Eulenbruch HP, Wrighton-Smith P, Enders G, Regnath T. Sensitivity of a new commercial enzymelinked immunospot assay (T SPOT-TB) for diagnosis of tuberculosis in clinical practice. Eur J Clin Microbiol Infect Dis. 2005;24(8):529-536.

51. Mazurek GH, LoBue PA, Daley CL, Bernardo J, Lardizabal AA, Bishai WR, Iademarco MF, et al. Comparison of a whole-blood interferon gamma assay with tuberculin skin testing for detecting latent Mycobacterium tuberculosis infection. JAMA. 2001;286(14):1740-1747.

52. Lee JY, Choi HJ, Park IN, Hong SB, Oh YM, Lim CM, Lee SD, et al. Comparison of two commercial interferongamma assays for diagnosing Mycobacterium tuberculosis infection. Eur Respir J. 2006;28(1):24-30.

53. Mazurek GH, Jereb J, Vernon A, LoBue P, Goldberg S, Castro K, Committee IE, et al. Updated guidelines for using Interferon Gamma Release Assays to detect Mycobacterium tuberculosis infection - United States, 2010. MMWR Recomm Rep. 2010;59(RR-5):1-25.

54. World Health Organization (WHO). Global tuberculosis report 2014. Geneva

55. Reichman LB. A scandalous incompetence...continued. Chest. 1998;113(5):1153-1154. 